\title{
Ensino e aprendizagem de conceitos algébricos com softwares: um panorama a partir de produções acadêmicas
}

Teaching and learning of algebra concepts with software: an overview from academic productions

Andressa Sanches Teixeira Sobrinho ${ }^{1}$ Maria Arlita da Silveira Soares ${ }^{2}$

\section{Resumo}

Este texto expõe como estão sendo abordadas questões relacionadas ao ensino e aprendizagem de conceitos algébricos com o auxílio de softwares de matemática dinâmica em pesquisas publicadas em periódicos brasileiros que publicam artigos da área da Educação Matemática. Fundamenta-se em estudos que abordam 0 desenvolvimento do pensamento algébrico e a teoria dos Registros de Representação Semiótica. Os dados foram produzidos e analisados a partir de Metanálise. Os resultados demonstram a predominância de propostas de ensino, na Educação Básica, principalmente no Ensino Médio. O foco da maioria das produções foi o conceito de função, com a utilização do software Geogebra. No que tange aos aspectos mais apontados na teoria dos Registros de Representação Semiótica, destacaram-se as transformações cognitivas, tratamento e conversão. Quanto às concepções da álgebra, percebeu-se que a maioria das pesquisas tratou de conceitos algébricos sem explicitar a importância das concepções da álgebra. Quanto ao pensamento algébrico, apenas uma produção investigou explicitamente suas características, as demais as apresentaram de forma implícita.

Palavras-chave: Álgebra. Pensamento algébrico. Softwares. Metanálise.

\section{Introdução}

Este texto é fruto de Teixeira (2016), pesquisa realizada pela primeira autora para compor seu Trabalho de Conclusão de Curso (TCC), no qual buscaram-se identificar e analisar os trabalhos publicados em periódicos brasileiros que publicam artigos da área da Educação Matemática que problematizam o ensino e a aprendizagem de conceitos algébricos e o uso de tecnologias (em particular, softwares), simultaneamente. A escolha dessa

\footnotetext{
${ }^{1}$ Mestranda em Educação nas Ciências pela Universidade Federal do Rio Grande do Sul (UFRGS), Professora da Educação Básica, andressaexatas2013@gmail.com.

${ }^{2}$ Doutora em Educação nas Ciências pela Unijuí/Ijuí, Professora da Universidade Federal do Pampa (UNIPAMPA), arlitasoares@gmail.com.
} 
temática foi pelo fato de que a álgebra é uma área da matemática que contribui para o desenvolvimento da criatividade, do raciocínio lógico e abstrato, das habilidades de gerar e comunicar ideias. Além disso, analisar as pesquisas já produzidas na área pode permitir uma compreensão diversificada das ideias da álgebra, através de múltiplos enfoques dados pelos educadores matemáticos brasileiros e de outros países.

A produção de dados do TCC deu-se a partir da análise de nove artigos, identificados em sete periódicos brasileiros que discutiam concomitantemente conceitos algébricos e a utilização de softwares. O recorte temporal para a seleção dos artigos compreendeu o período de 2010 a 2015. A análise dos dados indicou que os softwares contribuem para a aprendizagem de conceitos algébricos, em especial, o de função, por facilitarem a visualização e mobilização de diferentes representações matemáticas.

Considerando os limites da pesquisa supracitada e a importância de continuar as investigações sobre questões relacionadas ao ensino e aprendizagem de conceitos algébricos mediados por softwares, optou-se por ampliar o mapeamento das produções, considerando como novo recorte temporal as duas últimas décadas, bem como a quantidade de periódicos, de sete para oito. Assim, este texto apresenta o mapeamento realizado em oito periódicos que publicam artigos da área da Educação Matemática, no período de 2000 a 2015, para identificar e analisar pesquisas que problematizaram 0 ensino $e$ aprendizagem de conceitos algébricos e o uso de tecnologias (em particular, softwares), simultaneamente.

Para apresentar os dados produzidos no mapeamento, destaca-se inicialmente a fundamentação teórica acerca do ensino e aprendizagem da álgebra e da importância da utilização dos softwares nesse campo da matemática. Em seguida, expõe-se a metodologia utilizada para constituir as análises do mapeamento, as quais serão apresentadas na seção seguinte. Por fim, apontam-se as considerações finais. 


\section{Compreensões acerca do ensino e aprendizagem da álgebra}

O ensino da álgebra, geralmente, limita-se à manipulação de símbolos e expressões algébricas, em detrimento do desenvolvimento das capacidades de abstração e generalização. A linguagem algébrica é mais que a manipulação de símbolos e pode ser imprescindível na resolução de problemas. (PONTE; BRANCO; MATOS, 2009, VAN DE WALLE, 2009).

Para entender o processo de ensino e aprendizagem da álgebra, torna-se essencial aprofundar as discussões acerca das diferentes concepções desse campo e sua natureza. Usiskin (1995) apresenta diferentes concepções da álgebra, com base no entendimento das funções das letras em cada situaçãoproblema, a saber: Equação, Função, Estrutural e Aritmética Generalizada.

A concepção denominada Equação corresponde a resolver equações, nas quais as letras assumem a função de incógnitas ou constantes, tendo por finalidade simplificar e determinar expressões literais.

$\mathrm{Na}$ concepção chamada de Função, tem-se por objetivo analisar a relação entre variações. Assim, o intuito não é determinar o valor das letras que, nesse caso, não são incógnitas e sim argumentos (representam valores de um domínio) ou parâmetros (representam um número do qual dependem outros números), ou seja, o objetivo é verificar como as variáveis variam. (USISKIN, 1995).

A concepção Estrutural refere-se à escrita algébrica, isto é, as variáveis são objetos da álgebra abstrata, sem valor numérico ou representação gráfica. Nas tarefas que envolvem esta concepção, as letras serão manipuladas com a utilização de regras, como por exemplo, fatorar, simplificar expressões, reduzir termos semelhantes. Sublinha-se que, mesmo sendo abstratas, as manipulações devem ter significado e permitir conclusões.

$\mathrm{Na}$ Aritmética Generalizada, as variáveis são generalizadoras de modelos, ou seja, o modelo é estendido até abranger infinitas possibilidades. Segundo Usiskin (1995), a noção de variável como generalizadora é imprescindível para a modelagem matemática. 
Entende-se que, para construir a compreensão de conceitos e procedimentos algébricos, é importante um trabalho articulado entre essas quatro concepções, bem como considerar os aspectos relacionados ao desenvolvimento do pensamento algébrico. Isso porque o foco do ensino da álgebra, para Ponte, Branco e Matos (2009) e Van de Walle (2009), está no desenvolvimento do pensamento algébrico, pois este permite "utilizar modelos matemáticos na compreensão, representação e análise de relações quantitativas de grandezas e, também, de situações e estruturas matemáticas, fazendo uso de letras e outros símbolos". (BRASIL, 2018, p. 80).

Ao tratar de aspectos relacionados ao desenvolvimento do pensamento algébrico, Ponte, Branco e Matos (2009) descrevem as capacidades relacionadas a este pensamento. Para os autores,

O pensamento algébrico inclui a capacidade de lidar com expressões algébricas, equações, inequações, sistemas de equações e de inequações e funções. Inclui, igualmente, a capacidade de lidar com outras relações e estruturas matemáticas e usá-las na interpretação e resolução de problemas matemáticos ou de outros domínios. A capacidade de manipulação de símbolos é um dos elementos do pensamento algébrico, mas também é o "sentido de símbolo" (symbolsense), como diz Abraham Arcavi, que inclui a capacidade de interpretar e usar de forma criativa os símbolos matemáticos, na descrição de situações e na resolução de problemas. (PONTE; BRANCO; MATOS, 2009, p. 10).

Constata-se, na citação acima, que as capacidades relacionadas ao pensamento algébrico envolvem: a) compreender conceitos algébricos; b) estabelecer relações com outros campos da matemática (por exemplo, aritmética e geometria); c) manipular símbolos. Também, percebe-se que o desenvolvimento dessas capacidades está vinculado à resolução de problemas.

Ponte, Branco e Matos (2009), fundamentados nas ideias do pesquisador James Kaput e considerando a complexidade de definir pensamento algébrico, descrevem três vertentes fundamentais: a) representar: diz respeito à capacidade do estudante em utilizar diferentes sistemas de representação; b) raciocinar: dedutivamente e intuitivamente, refere-se a capacidade de relacionar as propriedades dos objetos matemáticos e generalizar as relações válidas para 
certa classe de objetos; c) resolver problemas: inclui modelar situações, bem como usar diversas representações.

Para Van de Walle (2009), o pensamento algébrico, ou raciocínio algébrico, está relacionado ao generalizar, a partir das operações e números; formalizar, através da utilização de símbolos; explorar, padrões e funções. Para o autor, o pensamento algébrico é essencial na matemática, tornando-a útil na vida cotidiana.

De acordo com os Parâmetros Curriculares Nacionais - PCN (BRASIL, 1998), a matemática deve possibilitar o entendimento sobre números e álgebra, mas não isoladamente de outros conceitos. Desse modo, a álgebra está relacionada à resolução de problemas, ao entendimento da linguagem simbólica, à descrição de modelos e à capacidade de utilizar a matemática na interpretação do mundo real.

Os entendimentos de Ponte, Branco e Matos (2009), Van de Walle (2009) e as diretrizes dos PCN (BRASIL, 1998) sobre desenvolvimento do pensamento algébrico evidenciam a capacidade de representar um problema - dado em língua natural - na linguagem matemática, em particular, na linguagem algébrica. Considerando que o desenvolvimento dessa capacidade é fundamental para a aprendizagem em matemática, recorre-se às ideias de Duval $(2011,2016)$ para compreender as especificidades desta área do conhecimento e da álgebra.

Segundo Duval (2011, 2016), em sua teoria dos Registros de Representação Semiótica, a especificidade da matemática, em relação às outras áreas do conhecimento, está no fato de que os objetos matemáticos são acessados somente por meio das diferentes representações semióticas. De acordo com o autor, a semiótica compreende registros e códigos. Um registro de representação semiótica é um sistema semiótico que desenvolve funções fundamentais para o funcionamento cognitivo consciente, "cujo poder para criar novas representações semióticas é ilimitado. O pensamento e a atividade matemática dependem totalmente da sinergia entre registros". (DUVAL, 2016, p. 4). Nesse sentido, Duval $(2011,2016)$ sugere que sejam utilizados na atividade 
matemática, pelo menos, dois tipos de registros de representação semiótica, e classifica-os em quatro tipos diferentes: língua natural, figuras geométricas, sistemas de escrita (numéricas, algébricas e simbólicas) e gráficos cartesianos.

$\mathrm{Na}$ atividade matemática, é importante distinguir dois tipos de transformações cognitivas: tratamento e conversão. O tratamento é uma transformação interna ao registro, por exemplo, a resolução de uma equação. "A conversão de uma representação é a transformação desta representação em uma representação de outro registro" (HENRIQUES; ALMOULOUD, 2016, p. 469), por exemplo, transformar a escrita de uma função (registro algébrico), para um gráfico cartesiano (registro gráfico). Além disso, quando uma conversão é realizada torna-se importante considerar o sentido, ou seja, determinar o registro de partida e de chegada. (DUVAL, 2011).

Diante deste contexto, percebe-se a importância do professor na elaboração de situações que exijam a mobilização das transformações cognitivas, destacadas por Duval $(2011,2016)$, em especial, a conversão e as três vertentes mencionadas por Ponte, Branco e Matos (2009), ou seja, busquem o desenvolvimento do pensamento algébrico dos estudantes.

Ao falar do desenvolvimento do pensamento algébrico, torna-se importante destacar o papel das tecnologias, em particular, dos softwares elaborados para o processo de ensino e aprendizagem da matemática. De acordo com Van de Walle (2009), a tecnologia deve ser considerada uma parte das ferramentas educacionais para a aprendizagem, podendo auxiliar no entendimento dos conceitos matemáticos. Para Ponte, Branco e Matos (2009), as tecnologias, em especial os softwares, possibilitam relacionar informações dadas algebricamente com as representações gráficas e em tabelas, assim como apresentam os objetos matemáticos com maior precisão e melhor visualização.

$\mathrm{Na}$ visão de Duval (2011), é importante destacar a contribuição dos softwares, em relação aos outros modos de produção de representações semióticas. $O$ autor afirma que devem-se analisar as tarefas cognitivas requeridas pela utilização destas ferramentas tecnológicas e sublinha que "eles constituem 
um modo fenomenológico de produção radicalmente novo, fundamentado na aceleração dos tratamentos" (DUVAL, 2011, p. 137). Neste sentido, obtém-se muito mais, em menos tempo, do que conseguiríamos à mão livre.

Em relação ao uso das tecnologias, em particular, os softwares para o ensino e aprendizagem de conceitos algébricos, Mcconnell (1995) defende que a adoção das tecnologias no ensino da matemática pode modificar a álgebra, tornando-a mais dinâmica, rica em variedade de aplicações, por exemplo, explorar a representação algébrica concomitantemente com a gráfica, entre outros. Também, afirma que as tecnologias desafiam os professores a provocarem nos estudantes a capacidade de julgamento, iniciativa e compreensão.

Historicamente, os primeiros softwares matemáticos disponíveis eram utilizados para trabalhar com situações que poderiam ser resolvidas por meio de algoritmos; em outras palavras, para exercitar treinamento e prática (HOUSE, 1995). Entretanto, hoje estão disponíveis softwares que podem influenciar as práticas em sala de aula.

Programas gráficos, por exemplo, fazem o que nenhuma lousa ou nenhum retroprojetor pode fazer, além de fornecerem aos Professores meios dinâmicos para demonstrar e explorar conceitos importantes como 0 comportamento de funções e seus gráficos. As planilhas eletrônicas tornam o Professor e o aluno capazes de empreender investigações do tipo "E se...?", como "E se você mudasse o argumento da função?" ou "E se você mudasse a hipótese para...?". (HOUSE, 1995, p. 6).

Sendo assim, as planilhas eletrônicas e os softwares integrados às atividades algébricas possibilitam um trabalho com gráficos e planilhas, que exige os conhecimentos algébricos, incentiva o trabalho de forma investigativa e também economiza tempo. A partir desse cenário acredita-se que a aprendizagem conceitual recebe mais atenção, visto que 0 tempo para 0 entendimento dos conceitos se expande e os procedimentos tornam-se significativos. Problematizações aproximando os estudantes de situações reais podem ganhar espaço através desses recursos. Além disso, entende-se que os softwares apresentam um avanço em relação à articulação da álgebra com a 
Geometria, pois os primeiros softwares restringiam-se aos objetos geométricos ou aos algébricos, sem articulá-los.

\section{Percurso metodológico}

O trabalho segue pressupostos da pesquisa qualitativa. Este tipo de pesquisa vem ganhando destaque, pois seu enfoque é relacionado à compreensão e discussão dos dados obtidos de maneira que o pesquisador problematize e não apenas apresente os resultados (BICUDO, 2014). Para a organização e análise dos dados buscaram-se orientações na Metanálise.

A Metanálise apresenta como característica principal o processo de reunir e reduzir dados, a partir do mapeamento de pesquisas, formando uma síntese. Entende-se que a Metanálise busca "integrar resultados de dois ou mais estudos, sobre um mesmo tema investigado" (BICUDO, 2014, p. 8). Portanto, trata-se de uma reflexão sobre o que foi investigado, na busca pelo sentido da investigação para o pesquisador, para o próprio tema investigado e para a região que se efetuou a pesquisa, procurando identificar tendências nas pesquisas analisadas.

Desse modo, foi realizado um mapeamento de trabalhos acadêmicocientíficos publicados nos periódicos, a saber: Boletim Gepem (UFRRJ ${ }^{3}$ ), Educação Matemática Pesquisa (PUC-SP ${ }^{4}$, Educação Matemática em Revista $\left(\right.$ SBEM-RS $\left.{ }^{5}\right)$, Zetetiké $\left(\right.$ UNICAMP $\left.^{6}\right)$, Bolema (UNESP ${ }^{7}$ ), Revemat (UFSC ${ }^{8}$ ), Acta $\left(U_{L B R A}{ }^{9}\right)$, e Vidya $\left(U F N^{10}\right)$. Optou-se por estes periódicos em função do Qualis (A1 e A2) na área de Ensino, com exceção do Boletim Gepem (Qualis B1), este por ser o periódico mais antigo na área da Educação Matemática. Além disso, todos possuem artigos disponíveis para download. Ainda, os dois periódicos que não tratam exclusivamente de pesquisas em Educação Matemática (Vidya e Acta)

\footnotetext{
${ }^{3}$ Universidade Federal Rural do Rio de Janeiro.

${ }^{4}$ Pontifícia Universidade Católica de São Paulo.

${ }^{5}$ Sociedade Brasileira de Educação Matemática - Rio Grande do Sul.

${ }^{6}$ Universidade Estadual de Campinas.

${ }^{7}$ Universidade Estadual Paulista - Júlio de Mesquita Filho.

${ }^{8}$ Universidade Federal de Santa Catarina.

${ }^{9}$ Universidade Luterana do Brasil - Canoas -RS.

${ }^{10}$ Universidade Franciscana - Santa Maria - RS.
} 
foram escolhidos por estarem vinculados a instituições do Estado da Federação em que a pesquisa foi realizada. Este critério também justifica a escolha do periódico Educação Matemática em Revista (SBEM-RS), além deste ter Qualis A2 na área de Ensino.

Para o mapeamento, foram elencados os seguintes descritores: pensamento algébrico, álgebra, tecnologias, softwares, Geogebra, Winplot, Graphmat. A escolha destes foi devido a intenção de identificar e analisar as pesquisas, publicadas em periódicos brasileiros que publicam artigos da área da Educação Matemática, que problematizassem o ensino e aprendizagem de conceitos algébricos e o uso de tecnologias (softwares), simultaneamente.

De acordo com os descritores escolhidos buscou-se, no texto dos artigos, que o trabalho versasse sobre a álgebra e o pensamento algébrico. Nessa primeira etapa, foram identificados vinte e oito artigos que correspondiam às expectativas iniciais. Posteriormente, foi realizada outra seleção a partir da releitura dos trabalhos, observando os resumos e, na sequência, a fundamentação teórica, para identificar a presença das tecnologias (softwares), restando doze que comtemplavam, concomitantemente, o primeiro e o segundo critério. O Quadro 1 apresenta o quantitativo de produções identificadas e os periódicos em que foram publicadas.

\begin{tabular}{|c|c|c|}
\hline Periódicos & 1 etapa & 2 etapa \\
\hline Boletim Gepem & 2 & 2 \\
\hline Educação Matemática Pesquisa & 7 & 1 \\
\hline SBEM - RS & 2 & 0 \\
\hline Zetetiké & 2 & 0 \\
\hline Bolema & 3 & 3 \\
\hline Revemat & 4 & 2 \\
\hline Acta & 4 & 1 \\
\hline Vidya & 4 & 3 \\
\hline Total & 28 & 12 \\
\hline
\end{tabular}

Quadro 1: Mapeamento de periódicos

Fonte: Elaborado para a pesquisa.

A identificação das produções, deu-se por A1, A2, ... A28 e mais detalhes dos artigos analisados estão disponíveis no Apêndice A. A partir destas doze 
produções, serão analisados os seguintes critérios: objetivos, participantes (destacando, níveis de ensino), conteúdos, softwares, transformações cognitivas (tratamento e conversão), concepções da álgebra e pensamento algébrico.

\section{Análise dos artigos mapeados que versam sobre conceitos algébricos e os softwares}

Os dados do Quadro 1 indicam que o número de produções acerca do tema investigado é maior nos periódicos Bolema e Vidya, seguido de Boletim Gepem e Revemat. Além disso, sublinha-se que as publicações que versam sobre conceitos algébricos e softwares concentraram-se no período de 2010 a 2015, sendo que 2013 foi o ano com maior número de publicações (cinco artigos).

A partir da análise dos objetivos das produções obteve-se: sete produções (A5, A6, A7, A20, A28, A29 e A30) com o intuito de elaborar e aplicar atividades com estudantes categorizadas como organização/desenvolvimento de propostas pedagógicas para vários encontros; três produções (A16, A19, A21) tratam da organização/aplicação de atividades em apenas um encontro e foram categorizadas como desenvolvimento de atividades; já duas produções (A13 e A18) tem como foco a elaboração de atividades que não foram aplicadas. Sublinha-se que as produções possuem objetivos semelhantes, ou seja, buscam elaboração e/ou desenvolvimento de atividades, visando minimizar as dificuldades dos participantes em relação a temas da álgebra. Além disso, as doze produções enfatizam o potencial da utilização de softwares como outra possibilidade para o ensino e aprendizagem de conceitos matemáticos.

Percebe-se, em geral, a preocupação dos autores em disponibilizar materiais didáticos para auxiliar práticas em sala de aula, bem como enfatizar a importância das tecnologias no ensino e aprendizagem da matemática. De acordo com os autores do artigo A16, "A importância da realização de tal pesquisa devese à necessidade de incluir o uso das tecnologias na disciplina de matemática e à carência de material didático voltado para esse fim" (A16, 2015, p. 209). 
A análise dos objetivos das pesquisas mapeadas também revela que todas envolveram participantes. O Quadro 2 apresenta os participantes, organizados por etapas de formação, a saber: Educação Básica, neste grupo estão as pesquisas que desenvolveram atividades com estudantes dos Anos Finais do Ensino Fundamental e Ensino Médio; Formação Inicial, nesta etapa estão as produções cujo público envolvia acadêmicos dos Cursos de Licenciatura em Matemática; Formação Continuada, neste grupo estão as pesquisas que trabalhou com professores de matemática que atuam na Educação Básica.

\begin{tabular}{|c|c|}
\hline Participantes & Artigos \\
\hline Educação Básica & A7; A16; A19; A 20, A21, A28, A29, A30 \\
\hline Formação Inicial & A6, A18 e A28 \\
\hline Formação Continuada & A5, A13 e A28 \\
\hline
\end{tabular}

Quadro 2: Participantes das pesquisas mapeadas

Fonte: Elaborado para a pesquisa.

Percebe-se que a maioria das pesquisas teve como participantes estudantes da Educação Básica, dentre os oito trabalhos neste nível, um foi desenvolvido no Ensino Fundamental e sete no Ensino Médio. Uma possível interpretação para este resultado refere-se ao fato de que o ensino da álgebra recebe maior atenção no Ensino Médio do que no Fundamental, pois este último prioriza o ensino de Números e Operações (PONTE, BRANCO, MATOS, 2009; VAN DE WALLE, 2009). Além disso, outro fator que pode impactar, neste resultado, é que as propostas curriculares para a Educação Básica sugerem o trabalho com recursos tecnológicos, pois estes podem proporcionar atividades experimentais que incentivam o desenvolvimento do pensamento matemático (BRASIL, 1998).

Conforme o Quadro 2, observa-se que apenas três artigos foram desenvolvidos com acadêmicos dos Cursos de Licenciatura em Matemática. $O$ documento intitulado "Subsídios para a discussão de propostas para os cursos de Licenciatura em Matemática", elaborado pela Sociedade Brasileira de Educação Matemática (SBEM), em parceria com a Sociedade Brasileira de Matemática (SBM), sobre a formação de professores, recomenda que os cursos promovam 
espaços-tempo para realização e análise de situações cujo objetivo é o desenvolvimento do pensamento algébrico por meio de atividades investigativas, resolução de problemas, entre outras opções metodológicas. (MUNIZ; SILVA, 2013).

Além disso, é importante proporcionar aos futuros professores subsídios para que saibam trabalhar com ferramentas tecnológicas (softwares). Segundo o documento supracitado, o professor de matemática ao incorporar as tecnologias na sala de aula poderá proporcionar ao estudante um trabalho investigativo, redimensionando sua prática e oportunizando novas condições para aprender matemática. Corroboram com estas ideias os autores do artigo A18, destacando que "há necessidade de repensar a formação dos professores de forma que sejam propiciados espaços de discussão das tecnologias na educação" (A18, 2015, p. 995).

Os dados do Quadro 2 indicam que apenas três trabalhos (A5, A13 e A28) envolveram a formação continuada, sendo que o artigo A28 desenvolveu atividades com os três níveis. De acordo com os autores desses trabalhos, tornase relevante a elaboração de materiais que orientem os professores a utilizarem softwares em suas aulas, pois estes profissionais só utilizarão estes recursos se estiverem seguros quanto ao seu funcionamento e convencidos do potencial que eles oferecem. Daí a necessidade de aproveitar o espaço da formação continuada para tal. Diante do número reduzido de trabalhos envolvendo a formação de professores, constata-se um campo promissor para novos trabalhos, visto que esta formação pode contribuir com 0 crescimento profissional $e$ consequentemente refletirá na aprendizagem dos estudantes.

O Quadro 3 expõe os conceitos/conteúdos matemáticos abordados nas pesquisas mapeadas, bem como os softwares escolhidos para a realização dos trabalhos. A análise dos dados do Quadro 3 permite afirmar que o conteúdo mais abordado nos artigos (A7, A13, A16, A20, A29 e A30) é função. Os argumentos para justificar esta tendência envolvem as dificuldades dos estudantes em relação a este conceito, a relevância do tema no currículo da Educação Básica, a 
importância das funções como linguagem das ciências, bem como a viável utilização de softwares para explorar as diferentes representações deste conceito.

No que tange aos tipos de funções abordadas nos seis artigos (Quadro 3), percebe-se, em quatro artigos, ênfase nas funções polinomiais e na exploração das ideias básicas do conceito de função, por exemplo: variáveis, correspondência, dependência, regularidades e generalização. O artigo A29 aborda funções trigonométricas, em especial, função seno e o artigo A30 tratou de funções logarítmicas e exponenciais, tendo como foco auxiliar os estudantes no reconhecimento de que uma é o inverso da outra.

\begin{tabular}{|c|c|c|c|c|c|c|c|}
\hline & $\begin{array}{c}\text { Trigono- } \\
\text { metria }\end{array}$ & $\begin{array}{c}\text { Deriva- } \\
\text { das }\end{array}$ & Funções & $\begin{array}{c}\text { Polinô- } \\
\text { mios }\end{array}$ & $\begin{array}{c}\text { Matrizes } \\
\text { e Det. }\end{array}$ & $\begin{array}{c}\text { Equa- } \\
\text { ções }\end{array}$ & $\begin{array}{c}\text { Isome- } \\
\text { trias }\end{array}$ \\
\hline GeoGebra & 1 & 1 & 2 & 1 & 1 & & 1 \\
\hline Graphmatica & & & 1 & & & & \\
\hline Winplot & & & 2 & & & & \\
\hline Scomax & & & & & & 1 & \\
\hline Applets & & & 1 & & & & \\
\hline
\end{tabular}

Quadro 3: Conceitos Matemáticos e softwares escolhidos

Fonte: Elaborado para a pesquisa.

Os artigos A16 e A20 mencionam a importância do conceito de função na relação da matemática com outras áreas do saber, em especial, as Ciências da Natureza (Física, Química e Biologia). No entanto, esta relação não foi identificada nas atividades propostas aos participantes das pesquisas. Embora 0 artigo A29 explore os conceitos envolvidos na função seno e o artigo A30 tenha como foco o estudo das funções logarítmicas e exponenciais, geralmente, utilizadas para interpretar fenômenos na área das Ciências da Natureza, as atividades escolhidas, nos dois, estão relacionadas apenas ao contexto matemático. Ressalta-se que poderiam ter associado, por exemplo, a função seno ao estudo das ondas sonoras e ter buscado situações como, meia vida de fármacos, decaimento radioativo, crescimento populacional de bactérias e fungos, para abordar conceitos das funções logarítmicas e exponenciais, explorando assim o contexto físico e matemático. 
Verifica-se, também no Quadro 3, que o software mais utilizado pelos autores foi o GeoGebra. A escolha do GeoGebra nos artigos (A5, A6, A18, A19, A20, A28, A29, A30), provavelmente, deve-se ao fato de que ele é "um software gratuito, com uma interface amigável, disponibilizando simultaneamente as representações algébrica e geométrica, além de possuir recursos de dinamicidade e movimentação" (A6, 2013, p. 429). Desse modo, destaca-se o potencial do software no ensino de funções, pois o mesmo pode contribuir no desenvolvimento do pensamento algébrico, em particular, na vertente representar, apontada por Ponte, Branco e Matos (2009), pois este recurso apresenta ao estudante diferentes representações de um mesmo objeto matemático.

Ainda em relação à escolha do software, os autores dos artigos (A5, A6, A18, A19, A20, A28, A29, A30) sublinham outros aspectos importantes considerados na escolha do GeoGebra, como, por exemplo, o potencial que o software apresenta para o levantamento e teste de conjecturas, a possibilidade de alcançar as generalizações e realizar demonstrações, bem como mobilizar uma variedade de representações. Percebem-se alguns destes aspectos nos trechos retirados dos artigos: "houve uma identificação positiva dos alunos com o GeoGebra, o que pode ser um indicativo de sua potencialidade para auxiliar os alunos no levantamento de conjecturas" (A28, 2011, p. 86); "O software permite que o aluno perceba diversas relações entre os objetos matemáticos, faça conjecturas e até mesmo generalize e formalize os resultados" (A19, 2013, p. 166). Nesse sentido, verifica-se que o software GeoGebra possibilita explorar as três vertentes (representar, raciocinar e resolver problemas), essenciais para o desenvolvimento do pensamento algébrico (PONTE, BRANCO, MATOS, 2009).

Ainda em relação ao uso de software, os autores dos artigos (A7; A13; A16; A 20, A29, A30), por exemplo, destacam que optaram por utilizar estas ferramentas tecnológicas para explorar funções, por acreditarem na exatidão, economia de tempo e também na capacidade investigativa que elas proporcionam aos estudantes. Verifica-se neste trecho, por exemplo, que as "contribuições do software GeoGebra no processo de aprendizagem foram salientadas por todos na 
precisão das medidas e na rapidez da realização das iterações fractais" (A20, 2013, p. 160). Estas ideias vão ao encontro do que afirma Duval (2011) em relação à exigência cognitiva no trabalho com os softwares, a precisão e economia de tempo que ele proporciona.

Em relação às transformações cognitivas, propostas por Duval (2011, 2016), para a aprendizagem matemática, apenas o artigo A30 discute sobre estas transformações, tratamento e conversão, pois foi o único que se fundamentou na teoria dos Registros de Representação Semiótica. No entanto, os demais artigos podem ser analisados a partir da ótica dos Registros de Representação Semiótica, pois defendem que o uso de softwares permite o trabalho com uma variedade de representações matemática.

Ainda sobre a conversão e o tratamento, o artigo A30, por exemplo, aponta algumas dificuldades dos estudantes, a saber: a conversão da representação do registro gráfico (registro de partida) para o algébrico (registro de chegada) e a elaboração das respostas por meio da língua natural. De acordo com Duval (2011), a primeira dificuldade apontada pode estar relacionada com a complexidade do reconhecimento das variáveis visuais pertinentes na representação gráfica associadas a valores numéricos da representação algébrica. Quanto à dificuldade com a mobilização do registro da língua natural, uma possível causa pode ser porque se trata de um registro que não é mais puramente matemático, existindo uma distância entre este registro e os outros. (DUVAL, 2011). Sendo assim, recomenda-se o uso de recursos como os softwares, para facilitar estas transformações auxiliando no reconhecimento das unidades de sentido matematicamente pertinentes no conteúdo das representações semióticas, podendo reduzir estas dificuldades.

Ao analisar os trabalhos quanto às diferentes concepções da álgebra, constata-se que o artigo A16 foi o único que trouxe de forma explicita definições para duas das quatro concepções da álgebra, neste caso, Funcional e Aritmética generalizada. Os autores explicam a dimensão funcional ao mencionar "quando analisamos a álgebra como o estudo das relações entre as grandezas, as 
variáveis representam valores do domínio de uma função ou números dos quais dependem outros números" (A16, 2015, p. 214) e referem-se à dimensão aritmética generalizada quando sublinham que "as variáveis são usadas como generalizadoras de informações numéricas" (A16, 2015, p. 214). Na sequência, estes pesquisadores enfatizam, implicitamente, a importância do desenvolvimento do pensamento algébrico com o estudo das funções, ao formular hipóteses, testar conjecturas e fazer generalizações, chegando a um modelo.

Verifica-se a preocupação em desenvolver o pensamento algébrico ao trabalhar com os softwares, mesmo que de forma implícita, nos textos mapeados. Pode-se fazer esta afirmação devido aos termos utilizados, na maioria dos artigos, como, por exemplo: procurar regularidades, formular, testar, justificar e provar conjecturas, refletir e generalizar. Cabe destacar que apenas um artigo tem como objetivo, de forma explícita, investigar as características do pensamento algébrico, representar, raciocinar e resolver problemas. Pode-se verificar esta afirmação nos seguintes recortes: "Saber representar e operar com expressões algébricas é importante para a compreensão do conceito e compreensão das aplicações de equações do $1^{\circ}$ grau" (A21, 2010, p. 90), "com relação a resolução de problemas a dificuldade mais explicitada estava relacionada com a dificuldade de estruturar/representar matematicamente as situações apresentadas" (A21, 2010, p. 92).

Conforme o exposto, os artigos apresentam, dentre os principais aspectos relacionados ao desenvolvimento do pensamento algébrico, o trabalho com análise de regularidades e generalizações. Desse modo, estes trabalhos estão de acordo com o que Van de Walle (2009, p. 287) destaca para o desenvolvimento de pensamento algébrico ou raciocínio algébrico, "formar generalizações a partir de experiências com números e operações, formalizar essas ideias com o uso de um sistema de símbolos significativo e explorar os conceitos de padrão e função". Além disso, as produções (A18 e A20), por exemplo, apostam na relação entre álgebra e Geometria, sendo esta uma estratégia adequada e sugerida por Van de 
Walle (2009), ao sublinhar que o pensamento algébrico deve ser incorporado em todas as áreas da matemática.

Desse modo, quanto ao pensamento algébrico, constatou-se que a maioria dos artigos apresenta ideias relacionadas ao pensamento de forma implícita. Em outras palavras, constata-se em algumas afirmações a busca por explorar o estabelecimento de relações, representar os objetos matemáticos de formas diferentes, modelar situações-problema, generalizar. Contudo, essas questões não são o foco principal das discussões apresentadas, pois a maioria dos autores dos textos busca organizar atividades que destaquem as potencialidades dos softwares, por isso, talvez as discussões acerca dos aspectos essenciais para o desenvolvimento do pensamento algébrico não estejam tão explícitas nas produções.

\section{Considerações finais}

A análise das produções permitiu concluir que a maioria das produções tinha por objetivo organizar e desenvolver propostas didático-pedagógicas, buscando minimizar dificuldades em relação à álgebra, assim como apresentar possibilidades de ensinar com o uso de softwares. Além disso, o maior número de artigos (8) teve como participantes estudantes da Educação Básica. Quanto à formação de professores, as produções dividiram-se entre três artigos desenvolvidos com a formação inicial e três artigos com a formação continuada.

Constatou-se que as produções valorizam a utilização dos softwares no estudo de conceitos matemáticos, em especial, funções, porque esses possibilitam o trabalho com diferentes representações do objeto matemático. $A$ escolha do conceito de função, em grande parte dos trabalhos, atribui-se à importância que possui no currículo da Educação Básica e pelas dificuldades apresentadas pelos estudantes. O software mais utilizado foi o Geogebra, por ser gratuito e de fácil acesso, bem como possibilitar a visualização concomitante de várias representações, o que contribui no desenvolvimento do pensamento algébrico. 
Em relação à teoria dos Registros de Representação Semiótica, observouse que apenas uma produção utilizou este aporte teórico. Contudo, a maioria enfatizou que os softwares auxiliam na visualização das várias representações matemáticas. Quanto às transformações cognitivas, tratamento e conversão, destaca-se que o software permite, por exemplo, que o estudante realize alterações no registro de representação algébrico e visualize, concomitantemente e instantaneamente, o que isso modifica no registro de representação gráfico, permitindo a análise das variáveis pertinentes de cada registro. Desse modo, entende-se que este recurso pode auxiliar a minimizar as dificuldades identificadas, principalmente, nas conversões entre representações semióticas.

No que tange as concepções da álgebra, apenas um artigo expõe de forma explícita a importância do reconhecimento destas concepções para o entendimento dos diferentes papéis que as letras podem assumir. Entretanto, salienta-se a importância das pesquisas que tratam de conceitos algébricos problematizarem acerca das várias concepções da álgebra.

Constatou-se, quanto ao desenvolvimento do pensamento algébrico, que a maioria das produções aponta que os softwares possibilitam analisar as situações, de modo a identificar as regularidades, generalizar e formalizar. Entretanto, verificou-se que o número de pesquisas que buscam relacionar a utilização de softwares com questões que tratam do desenvolvimento do pensamento algébrico, em geral, ainda são poucas. As produções analisadas apresentaram mais referências relacionadas à utilização dos softwares, preocupando-se em justificar a importância do trabalho com estas ferramentas, do que em relação ao desenvolvimento do pensamento algébrico e o que ele proporciona para o entendimento da matemática.

Além disso, pode-se perceber que, embora exista a recomendação de propostas curriculares para trabalhar com o desenvolvimento do pensamento algébrico desde os Anos Iniciais do Ensino Fundamental, as produções que fizeram parte deste mapeamento não tiveram como participantes este público. 
Uma possível interpretação para este resultado é que o trabalho com a álgebra nos anos iniciais ainda é restrito em função da ênfase em números e operações.

Sendo assim, sugere-se, que seja estabelecida uma relação entre o desenvolvimento do pensamento algébrico e as concepções da álgebra na estruturação de propostas de ensino que utilizem softwares de matemática Dinâmica para seu desenvolvimento.

\section{Referências}

BICUDO, Maria Aparecida Viggiani. Meta-análise: seu significado para a pesquisa qualitativa. Revista Eletrônica de Educação Matemática - REVEMAT, Florianópolis, Ed. temática, v. 9, p. 7-20, Jun. 2014.

BRASIL. Ministério da Educação e do Desporto. Parâmetros Curriculares Nacionais Matemática 5ª a 8aㅗ. série. Brasília: SEF, 1998.

BRASIL. Ministério da Educação. Sistema de Avaliação da Educação Básica. Brasília: SAEB, 2018.

DUVAL, Raymond. Ver e Ensinar a Matemática de outra Forma: Entrar no Modo Matemático de Pensar: os Registros de Representações Semióticas. Organização Tania M. M. Campos. Tradução Marlene Alves Dias. 1. ed. São Paulo: PROEM, 2011.

DUVAL, Raymond et al. Questões epistemológicas e cognitivas para pensar antes de começar uma aula de matemática. Revemat: Revista Eletrônica de Educação Matemática, v. 11, n. 2, p. 01-78, 2016.

HENRIQUES, Afonso; ALMOULOUD, Saddo Ag. Teoria dos registros de representação semiótica em pesquisas na Educação Matemática no Ensino Superior: uma análise de superfícies e funções de duas variáveis com intervenção do software Maple. Ciência \& Educação, Bauru, vol.22, n.2, p.465-487, 2016.

HOUSE, Peggy A. álgebra: ideias e questões, 1995. In: As ideias da álgebra. COXFORD, Arthur F.; SHULTE, Albert. P. (ORG.) Tradução: DOMINGUES, H. H. São Paulo: Atual, 1994.

MCCONNELL, J. Uso de computadores e calculadoras no aprendizado da álgebra, 1995. In: As ideias da álgebra. Organizadores: COXFORD, Arthur. F.; SHULTE, Albert. P. Tradução: DOMINGUES. H. H. São Paulo: Atual, 1994.

MUNIZ, Cristiano Alberto; SILVA, Hilário Alencar da. A Formação do Professor de Matemática no Curso de Licenciatura: Reflexões Produzidas pela Comissão Paritária SBM/SBEM. Sociedade Brasileira de Educação Matemática, n. 21,p. 1-42, 2013. 
PONTE, João Pedro da; BRANCO, Neusa; MATOS, Ana. álgebra no Ensino Básico. Ministério da Educação de Portugal, 2009.

TEIXEIRA, A. S. O desenvolvimento do pensamento algébrico e os softwares de matemática dinâmica: metanálise de produções brasileira. 2016. 30f. Trabalho de Conclusão de Curso - UNIPAMPA, Caçapava do Sul, 2016.

USISKIN, Zalman. Concepções sobre a álgebra da escola média e utilizações de variáveis, 1995. In: As ideias da álgebra. Organizadores: COXFORD, A. F.; SHULTE, A. P. Tradução: DOMINGUES. H. H. São Paulo: Atual, 1994.

VAN DE WALLE, Jonh. A. Matemática no Ensino Fundamental: formação de professores e aplicação em sala de aula. Tradução: COLONESE, P. H. 6. ed. Porto Alegre: Artmed, 2009.

\section{Apêndice A}

\begin{tabular}{|c|c|c|c|}
\hline & Título & Autores & Periódico \\
\hline A5 & $\begin{array}{c}\text { A Gênese Instrumental na Interação } \\
\text { com o GeoGebra: uma proposta para a } \\
\text { formação continuada de professores de } \\
\text { Matemática }\end{array}$ & $\begin{array}{l}\text { Celina A. A. P. Abar; } \\
\text { Sergio Vicente Alencar }\end{array}$ & Bolema \\
\hline A6 & $\begin{array}{l}\text { Atividades Investigativas de Aplicações } \\
\text { das Derivadas Utilizando o GeoGebra }\end{array}$ & $\begin{array}{l}\text { Daniele C. Gonçalves; } \\
\text { Frederico da Silva Rei }\end{array}$ & Bolema \\
\hline A7 & $\begin{array}{c}\text { Ensino de Funções Polinomiais de } \\
\text { Grau Maior que Dois Através da } \\
\text { Análise de seus Gráficos, com Auxílio } \\
\text { do Software Graphmatica }\end{array}$ & $\begin{array}{l}\text { Clóvis José Dazzi; } \\
\text { Maria Madalena Dullius }\end{array}$ & Bolema \\
\hline A13 & $\begin{array}{c}\text { Ideias básicas de função no 9o ano do } \\
\text { ensino fundamental: uma sequência de } \\
\text { atividades com o auxílio do software } \\
\text { Winplot }\end{array}$ & Karina de Oliveira Castro & Revemat \\
\hline A16 & $\begin{array}{l}\text { Explorando os coeficientes da função } \\
\text { quadrática por meio do software } \\
\text { Winplot: Uma experiência com alunos } \\
\text { do } 2^{\circ} \text { ano do Ensino Médio }\end{array}$ & $\begin{array}{l}\text { Mauricio Ramos Lutz; } \\
\text { Aline Silva de Bona }\end{array}$ & Revemat \\
\hline A18 & $\begin{array}{c}\text { Tecnologias digitais no ensino: } \\
\text { discussões a partir de propostas } \\
\text { desenvolvidas por licenciandos } \\
\text { envolvendo polinômios }\end{array}$ & $\begin{array}{l}\text { Maria Ivete Basniak; } \\
\text { Dirceu Scaldelai; } \\
\text { Celine Maria Paulek; } \\
\text { Natali Angela Felipe }\end{array}$ & $\begin{array}{l}\text { Educação } \\
\text { Matemática } \\
\text { Pesquisa }\end{array}$ \\
\hline A19 & $\begin{array}{l}\text { Investigação matemática com o } \\
\text { GeoGebra: um exemplo com matrizes } \\
\text { e determinantes }\end{array}$ & $\begin{array}{l}\text { Duelci A. de Freitas Vaz; } \\
\text { Elivanete Alves de Jesus }\end{array}$ & $\begin{array}{l}\text { Boletim } \\
\text { Gepem }\end{array}$ \\
\hline A20 & $\begin{array}{c}\text { Construção de fractais usando o } \\
\text { software GeoGebra }\end{array}$ & $\begin{array}{l}\text { Teresinha A. F. Padilha; } \\
\text { Maria Madalena Dullius; } \\
\text { Marli Teresinha Quartieri }\end{array}$ & $\begin{array}{l}\text { Boletim } \\
\text { Gepem }\end{array}$ \\
\hline A21 & Características do pensamento & Claudia L. O. Groenwald; & Acta \\
\hline
\end{tabular}




\begin{tabular}{|c|c|c|c|}
\hline & $\begin{array}{l}\text { algébrico de estudantes do Ensino } \\
\text { Médio com equações do } 1^{\circ} \text { grau }\end{array}$ & Ednei Luis Becher & \\
\hline A28 & $\begin{array}{c}\text { Explorar e comunicar ideias sobre } \\
\text { isometrias }\end{array}$ & $\begin{array}{c}\text { Maria Auxiliadora Lage } \\
\text { Maria Clara R. Frota }\end{array}$ & Vidya \\
\hline A29 & $\begin{array}{l}\text { Explorando modelos matemáticos } \\
\text { trigonométricos a partir de applets }\end{array}$ & $\begin{array}{l}\text { Marlizete Franco da Silva; } \\
\text { Maria C. Rezende Frota }\end{array}$ & Vidya \\
\hline A30 & $\begin{array}{l}\text { Análise das estratégias utilizadas pelos } \\
\text { estudantes no estudo de funções } \\
\text { logarítmicas e exponenciais }\end{array}$ & $\begin{array}{l}\text { Adriana Tiago dos Santos; } \\
\text { Barbara Lutaif Bianchini }\end{array}$ & Vidya \\
\hline
\end{tabular}

Quadro 4: Identificação dos artigos analisados

Fonte: Elaborado para a pesquisa. 